\title{
High dose right unilateral was as effective as bilateral electroconvulsive therapy for major depression
}

Sackeim HA, Prudic J, Devanand DP, et al. A prospective, randomized, double-blind comparison of bilateral and right unilateral electroconvulsive therapy at different stimulus intensities. Arch Gen Psychiatry 2000 May;57:425-34.

QUESTION: In patients with major depressive disorder (MDD), how do different dosages of right unilateral (RUL) electroconvulsive therapy (ECT) compare with high dosage bilateral (BL) ECT for antidepressant response and cognitive side effects?

\section{Design}

Randomised \{allocation concealed*\} $\uparrow$, blinded (patients and outcome assessors), * controlled trial with 1 week of follow up after the last ECT session.

\section{Setting}

New York State Psychiatric Institute, New York, USA

84 patients (96\% inpatients) who had MDD with a score of $\geq 18$ on the 24 item Hamilton Rating Scale for Depression (HRSD). Exclusion criteria were history of functional psychosis or rapid cycling bipolar illness; neurological abnormality; substance abuse in the past year; ECT in the past 6 months; or severe medical illness. 80 patients (mean age $57 \mathrm{y}, 64 \%$ women) completed the study.

\section{Intervention}

At the first ECT session patients' seizure threshold (ST) was quantified and they were allocated to 1 of 4 groups with 20 patients each: RUL ECT with stimulus intensity at either 1.5 (low dose), 2.5 (moderate dose), or 5.0 (high dose); or BL ECT at 2.5 (high dose) times the initial ST.

\section{Main outcome measures}

Initial antidepressant response (decrease of $\geq 60 \%$ in HRSD scores and a score of $\leq 16$ at 1 to $2 \mathrm{~d}$ after the last treatment). Final responders maintained response 1 week

\section{Patients}

after the final treatment. Cognitive side effects were evaluated using anterograde and retrograde memory tests.

\section{Main results}

High dose RUL and BL ECT initial and final responder rates did not differ. Compared with BL ECT, fewer RUL ECT low and moderate dosage group patients had initial response $(p=0.002)$ (table). Fewer RUL ECT group patients had final response than did BL ECT group patients $(p=0.03)$ (table). Cognitive disturbance was greatest in the BL ECT group. The rates for disorientation immediately after treatment lasting $>90$ minutes and the extent of word recall or recognition were greatest with $\mathrm{BL}$ ECT than with any RUL ECT dosage $(p=0.002$ and $\mathrm{p}=0.03$, respectively, for difference between BL ECT and all RUL ECT groups). 2-7 days after final treatment BL ECT resulted in greater impairment than any RUL ECT dosage on several cognitive measures. Cognitive differences persisted 2 months after completion of ECT.

\section{Conclusion}

In patients with major depression, markedly suprathreshold right unilateral electroconvulsive therapy (ECT) was as efficacious as high dose bilateral ECT for antidepressant response and had fewer cognitive side effects.

*See glossary.

$\dagger$ Information provided by author

Right unilateral electroconvulsive therapy (RUL ECT) v bilateral (BL) ECT for antidepressant response in major depression $\neq$

\begin{tabular}{llllll}
\hline Outcome time & Comparison & Rates & ABR (95\% Cl) & RBR (Cl) & NNH (Cl) \\
\hline \multirow{2}{*}{1 to 2 days (initial response) } & Low dose RUL $v$ BL ECT & $35 \% v 80 \%$ & $45 \%(14$ to 68$)$ & $56 \%(22$ to 78$)$ & $3(2$ to 8$)$ \\
\hline & Moderate dose RUL $v$ BL ECT & $45 \% v 80 \%$ & $35 \%(4.7$ to 60$)$ & $44 \%(7.9$ to 69$)$ & $3(2$ to 20$)$ \\
\hline & High dose RUL $v$ BL ECT & $80 \% v 80 \%$ & $0 \%(-26$ to 26$)$ & $0 \%$ & Not significant \\
\hline 1 week (final response) & Low dose RUL $v$ BL ECT & $35 \% v 65 \%$ & $30 \%(-1.4$ to 56$)$ & $46 \%(-2$ to 74$)$ & Not significant \\
\hline & Moderate dose RUL $v$ BL ECT & $30 \% v 65 \%$ & $35 \%(3.7$ to 60$)$ & $54 \%(8.1$ to 79$)$ & $3(2$ to 25$)$ \\
\hline & High dose RUL $v$ BL ECT & $65 \% v 65 \%$ & $0 \%(-29$ to 29$)$ & $0 \%$ & Not significant \\
\hline
\end{tabular}

$\ddagger A B R=$ absolute benefit reduction; RBR=relative benefit reduction. Other abbreviations defined in glossary; $\mathrm{ABR}, \mathrm{RBR}, \mathrm{NNH}$, and $\mathrm{Cl}$ calculated from data in article.

\section{COMMENTARY — continued from previous page}

McCall et al compared titrated moderately suprathreshold with fixed high dose RUL ECT. Previously published trials have reported RUL ECT efficacy and memory effects to be sensitive to stimulus dosing above ST. ${ }^{1}$ McCall et al confirmed these findings in their study as retrospective analysis of the fixed high dose RUL ECT group showed that treatments at dosage intensities from 3.15 to 5.04 times ST were much less effective than those at intensities from 8.4 to 12.6 times ST. Differences detected in adverse effects were apparent 1-2 days after the ECT course at a dosage of 3.15 to 5.04 times ST, but were dramatically worsened at a dosage of 8.4 to 12.6 times ST. Such worsening effects on immediate memory paralleled improvement in antidepressant efficacy. As with the results of the study by Sackeim et al, clinicians will have to balance the antidepressant benefit of high dose RUL ECT against increased memory impairment.

The BL $v$ RUL ECT controversy remains unresolved. Readers should be cautious about adopting a recommendation for RUL ECT at 6 times ST in their clinical practice, even for depressed patients. Short term cognitive side effects are less likely, but problems with small sample size and choice of BL ECT dosage intensity in the study by Sackeim et al mean that important clinical questions remain about their comparative efficacy. The results from McCall et al suggest that, as assessed, RUL ECT is too inefficient for routine use and the reduced memory impairment is of limited clinical significance. My recommendation for practitioners is that BL ECT at 1.5 times the estimated or measured ST remains the clinical standard.

Max Fink, MD State University New York at Stony Brook Long Island, New York, USA

1 Sackeim HA, Prudic J, Devanand DP, et al. Effects of stimulus intensity and electrode placement on the efficacy and cognitive effects of electroconvulsive therapy. $N$ Engl J Med 1993;328:839-46. 